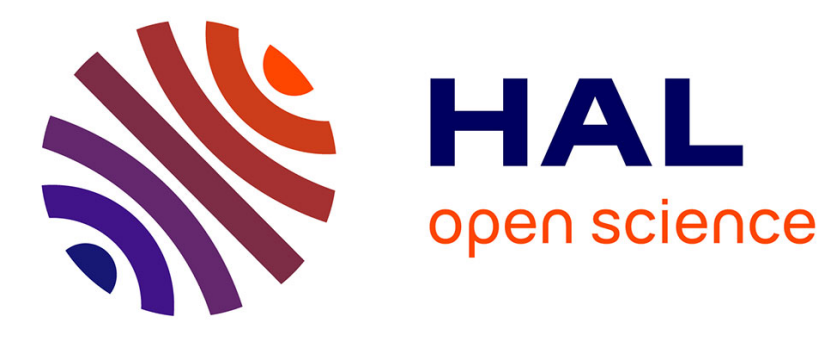

\title{
Conceptualization of genetic disease
}

Catherine Dekeuwer

\section{To cite this version:}

Catherine Dekeuwer. Conceptualization of genetic disease. Thomas Schramme; Steven Edward. Handbook of the philosophy of medicine, Springer, pp.1-18, 2017, Springer reference, 978-94-0178687-4. 10.1007/978-94-017-8706-2_81-1. hal-01791750

\section{HAL Id: hal-01791750 https://univ-lyon3.hal.science/hal-01791750}

Submitted on 27 Sep 2020

HAL is a multi-disciplinary open access archive for the deposit and dissemination of scientific research documents, whether they are published or not. The documents may come from teaching and research institutions in France or abroad, or from public or private research centers.
L'archive ouverte pluridisciplinaire HAL, est destinée au dépôt et à la diffusion de documents scientifiques de niveau recherche, publiés ou non, émanant des établissements d'enseignement et de recherche français ou étrangers, des laboratoires publics ou privés. 
Conceptualization of Genetic Disease Handbook of the Philosophy of Medicine (T. Schramme and S. Edwards eds.), Springer, $2017: 1-18$

\section{Abstract}

The expression "Genetic Disease " suggests the idea that one or more genes cause a disease. Since the identification of the so-called "disease-causing genes", a lot of genetic variants associated with common diseases like diabetes or cancers have being identified. But if the involvement of genetics factors in the development of a condition justifies labeling it "genetic", the definition of genetic disease risks becoming trivial. Indeed, in this case, all human diseases would be genetic, since virtually every medical condition, disease susceptibility or response to treatment is caused, regulated or influenced by genes. Moreover, genes alone are not responsible for the development of diseases: their etiology is complex with many factors interacting with each other. So when is giving primary importance to genetic factors justified? The history of medicine may help us see which biological and technological advances have influenced conceptualization of genetic disease, while philosophical analyses of the causal selection problem may help us better understand how one might justify claims that the genes are the main causes of certain diseases. The last part of the article examines interactionist attempts to deal with genetic factors involved in human diseases and particularly network medicine.

\section{Introduction}

The expression "Genetic Disease » suggests the idea that one or more genes cause a disease. For example, Phenylketonuria, cystic fibrosis, Huntington disease or certain forms of breast and ovarian cancers are generally considered to fit this definition, which arose from the localization and identification of the so-called "disease-causing genes" at the end of the twentieth century.

The history of genetic medicine however, which began with an enthusiastic program for discovering the disease-causing genes, now seems to be stumped by the embarrassing observation that all diseases might be caused by some genes. A lot of genetic variants associated with common diseases have been identified, including the sporadic form of Alzheimer's disease, obesity, diabetes, sporadic cancers, antisocial personality disorders, and even tuberculosis. Consulting the catalogue of human genes and genetic disorders (Online Mendelian Inheritance in Man) makes one even more puzzled, as diseases linked to mitochondrial DNA mutations, epigenetic modifications and even congenital disorders are referenced. This has led some scholars to ask whether cancer or tuberculosis could be labeled "genetic diseases" and raises the problem of the triviality of the concept. Extending the concept of genetic disease empties the term of its specificity and so no longer conveys any useful information. Moreover, genes alone are not responsible for the development of diseases: their etiology is complex with many factors interacting with each other. This raises a key question: when is giving primary importance to genetic factors justified?

In order to set some guides in what looks like a fuzzy landscape, the first section starts with the history of medicine, so as to recount the biological and technological advances that have influenced the conceptualization of genetic disease, as well as drawing our attention to some potential shifts in 
that conceptualization. The second section will then consider philosophical analyses of the causal selection problem with a view to discussing the ways in which one may justify the claim that the genes are the main causes of certain diseases. The last section will turn to some interactionist contemporary developments of biology and medicine that enlighten the puzzling use of the concept of genetic disease.

\section{1 - From Predictive to Personalized Medicine}

In the 1970s, French Nobel Prize J. Dausset developed the concept of predictive medicine. The discovery of correlations between histocompatibility antigens and susceptibility to illness, the hypothesis that a set of genetic factors determines for each individual her susceptibility to common diseases like cancers, and the first advances in the attempt to map the human genome supported the idea that 21st century medicine will be predictive. Intended for healthy individuals, predictive medicine was conceived as the practice of determining whether their susceptibility to specific diseases was increased and proposing appropriate measures of prevention.

The similarity of Dausset's project with the means and goals of contemporary genomic medicine is striking. The hope that medicine will prevent common diseases like cancers, diabetes, asthma, mental illnesses or heart diseases supported major biological projects such as the Human Genome Project (HGP) and Genome-wise association studies (GWAS). The National Human Genome Research Institutes presentation of the GWAS project states for example: "the impact on medical care from genome-wide association studies could potentially be substantial (...) In the future, after improvements are made in the cost and efficiency of genome-wide scans and other innovative technologies, health professionals will be able to use such tools to provide patients with individualized information about their risks of developing certain diseases. The information will enable health professionals to tailor prevention programs to each person's unique genetic makeup. In addition, if a patient does become ill, the information can be used to select the treatments most likely to be effective and least likely to cause adverse reactions in that particular patient" (https://www.genome.gov/20019523, accessed 20 July 2015). This project, often called "personalized medicine", is today at the forefront of much medical researches.

The history of the changes that occurred since the 70s can be described as a two phase process. Some distinctions between genetic and genomic medicines, genetic causes and genetic factors, monogenic and common diseases can be found in scientific literature. For example, some researchers have a preference for the expression "genomic medicine" over "genetic medicine", for it implies a focus on medicine that uses new technological methods of genomic data acquisition and analysis to study the complex interaction between genetic and environmental factors in common diseases and, as such, it is clearly distinct from the attempt to study Mendelian monogenic diseases. However, most of the time, there are no such clear distinctions. A brief survey of the history of medicine sheds light on how anarchical the use of the concept of genetic disease is today.

\subsection{Monogenic diseases are not simple}


In the 1980s, the identification of so-called "disease-causing genes" led the predictive medicine project to be temporarily put aside. Researchers carried out linkage analyses in order to map the genes for monogenic diseases such as thalassemia, Huntington disease or cystic fibrosis. Even if known environmental factors (for example altitude in the case of thalassemia) or unknown phenomena play a part in the age of onset of the symptoms or their severity, the penetrance of these monogenic diseases is almost 1: almost all the individuals carrying a particular variant of a gene (the so-called "genetic mutation") also express the pathological phenotype. At the same time, molecular medicine was also rapidly expanding. Following the "central dogma" of molecular biology, it sought to explain why a given genetic change should result in a particular clinical phenotype, how a change in a particular DNA sequence could modify the quantity or function of the gene's product and why this change is pathogenic for a cell, a tissue or a stage of development.

As a small proportion of breast and ovarian cancers are inherited in an autosomal dominant manner, BRCA1 and BRCA2 genes were localized in 1990 and 1994 using linkage analysis. The presence of BRCA1 or BRCA2 specific variants significantly increases the risk of developing such cancers, but the penetrance of the pathological phenotype is incomplete: a proportion of individuals carrying the identified variants will never develop the symptoms. For instance, in 2007, the cumulative risk of developing breast cancer for a person carrying a BRCA1 mutation at age 70 years was estimated at $57 \%$, while the risk is about $10 \%$ in the general population (Chen et al., 2007). The concept of genetic predisposition was born, referring to a highly increased risk of developing a disease associated with specific genetic variants. The molecular effects of the BRCA1 and BRCA2 variants began to be studied.

The localization and identification of some disease-causing genes and genetic predispositions led to the rapid set up of specific medical practices, such as genetic counseling, aimed at helping individuals to make autonomous decisions regarding genetic testing. As more and more genetic presymptomatic and prenatal tests were available, raising important ethical questions, several countries chose to supervise genetic medicine by specific legislations. Genetic medicine is today a normalized practice based on the knowledge of very high correlations between the presence of specific genetic variants and the apparition of certain diseases in a family. Increasingly, the specific molecular effects of these variants are also being identified.

Recalling this history underlines three characteristics of the concept of genetic disease. (1) A genetic disease is inherited according to Mendel's laws. (2) The development of symptoms is highly correlated with the presence of specific genetic variants whose knowledge supports genetic testing. (3) One can explain the contribution of the variants to the molecular transformations responsible of the symptoms. These three characteristics are not necessary together for labelling a disease "genetic" but they work as landmarks when reflecting on how conceptualize genetic disease.

Things became more difficult as knowledge of the relationship between genotype and phenotype developed further. For example, one used to consider phenylketonuria as a monogenic disease caused by some specific variations in the PAH gene. But, in absence of PAH mutations, an abnormal $\mathrm{BH} 4$ protein may result from other genetic variations and also cause the specific symptoms of phenylketonuria. Besides conceptualizing this phenomenon, called "genetic heterogeneity", the hard tasks are to understand the phenomenon of incomplete penetrance as well as why - considering the same genetic variant - symptoms may be more or less severe, and why the expressivity of diseases is variable, some patients developing one set of symptoms whereas others suffer of a different set. 
Some studies (see Friebel et al. 2014) have tried to show the influence of environmental factors on the development of symptoms, such as pregnancies or history of breast feeding for breast and ovarian cancers. Genetic factors and genetic regulation may also explain the phenotypic variations of the same monogenic disease. For example, the CFTR gene was established in 1989 as the "cystic fibrosis causing gene", but variations in lung and digestive symptoms correlate poorly with variants of the CFTR gene as well as with environmental factors. Considering that the phenotype may be polygenic (that is, modified by the presence or absence of genetic variants other than the CFTR gene), "modifier genes" have been identified, which influence the phenotype. Cumulative genetic effects, genetic regulation and the functional role of specific genetic variants in the control of infection, immunity or inflammation are all working hypotheses aiming to account for the phenotype-genotype correlation in cystic fibrosis disease (Dipple and McCabe, 2000; Badano and Katsanis, 2002).

Disease-causing genes, as well as genetic predispositions, do not work in an "all or nothing" way. Understanding the variable expressivity of monogenic diseases, the differences in the severity of symptoms, or in the timing of their appearance requires the study of several genetic factors that interact with each other, as well as of the interactions between genetic and environmental factors. It also requires the understanding of their involvement in the biological pathways that lead some people to be sick. In other words, the apparent simplicity of the concept of genetic disease has given way to an appreciation of its complexity.

\subsection{The black-box strategy of Genome-Wise Association Study}

Association studies led to the identification of many genetic variants statistically correlated to common diseases (see Visscher et al., 2012). As the knowledge of the mode of inheritance of the disease is not necessary to complete association studies, they are particularly useful for studying common diseases. The recent GWAS are based on the "common disease - common variant" hypothesis (Reich and Lander 2001), which states that common diseases are attributable in part to genetic variants very common in the population. These variants are not sufficient to cause a condition but they are statistically more frequent in the affected population than in the non-affected one. The strategy of GWAS is to study large populations of healthy vs. affected individuals, and find some differences in the frequency of markers in individuals who are or are not affected by the condition (the most commonly used markers are called SPNs). Thus, nothing is known about the biological effect of the genetic component highlighted in this way: GWAS, like other association studies formerly used, is a "black-box strategy". For this reason, a genetic variant that has been identified by association study cannot be considered as a cause of the disease. The concept of genetic factor thus, turns out to be quite empty, for it amounts only to a specific SNP statistically correlated to a disease that confers at the best a slightly increased relative risk in comparison to the general population. Problems raised by these association studies like size and choice of populations, reproducibility of the study or the threshold of significance of the correlations are now well known (Visscher et al., 2012).

GWAS also raises some additional difficulties. First, it gives no clue as to the genetic architecture of common diseases, i.e., the way genotype and phenotype are related. Moreover, these common genetic variants confer only a relatively small increment in risk and explain only a small proportion of 
heritability. For example, even if the estimated heritability of a complex trait is about $80 \%$, the genetic variations associated with this trait generally explain only about $5 \%$ of that heritability, despite studies of thousands of people. This phenomenon, called "missing heritability", led some researchers to state a new hypothesis: "common disease - rare variant". According to that hypothesis, the effects of low frequency variants of intermediate effect and the interaction between several genes or the effect of epigenetic factors might contribute to the explanation of missing heritability (see Manolio, 2009).

However, the main limitation of association studies remains their black-box strategy, which makes it necessary to add further biological investigations about the potential functions of the DNA regions identified. Actually, it is also important to note that association studies are not the only example of this limited strategy. For instance, inheritance of Huntington Disease was first associated with a genetic marker and the first genetic tests were set up without any knowledge of the sequence and the function of the gene involved in the disease (Bates, 2005). To take another example, the history of the identification of the CFTR gene shows how the researchers confined a locus by means of a genetic marker, picked out candidate genes and sequenced them. This strategy of "reverse genetic" led them to select the CFTR gene, whose function was congruent with biological and pathological evidence (see Dekeuwer, 2015). Today, GWAS is frequently opposed to candidate gene approach since its "wide" strategy is not compatible with the selection of pre-specified genes of interest, i.e., candidate genes. However, when conducting medical research, it is necessary to complete the identification of a set of SNP markers correlated with a particular condition with the study of the potential biological pathways in which the related DNA region might be involved.

Thus the contemporary, somehow anarchical use of the concept of genetic disease is neither only the consequence of some still more complex analyses of the human genome nor the reflection of the extension of our knowledge of the genetic determinants of diseases. The developments of classical Mendelian genetics also cast doubts on the concepts. As this first part takes us back ceaselessly to the complexity of the interactions involved in pathological pathways, it seems now necessary to raise this question: why should genetic factors be the most important?

\section{Genes that "make the difference"}

The etiology of biological traits is extremely complex and involves a large number of causally relevant factors that interact with each other. The causal selection problem is the problem of how to select the cause "that makes the difference" and conceptualize it. Some philosophers (Hesslow, 1984 and 1988; Norell, 1984; Gifford, 1990; Gannett, 1999; Magnus, 2004; Smith, 2007; Dekeuwer, 2015) have tried to give critical assessment of the criteria that may resolve the causal selection problem. However, since all these criteria show insufficiencies, and as it is difficult to envision a trait in which genes are not involved, it seems impossible to prevent the concept of genetic disease becoming absolutely trivial. This section resumes some arguments that support the fact that genes could make the difference and discusses the consequences of an unjustified extension of the concept of genetic disease.

\subsection{Genes as specific causes}


The first strategy to solve the causal selection problem tries to specify the causal relationship between gene and phenotype. Criteria of necessity, sufficiency and directness have been discussed, but the strongest criterion is the one of specificity. As F. Gifford argues, in order to be considered genetic, "a trait must be described or individuated in such a way that it is properly matched to what the gene causes specifically" $(1990,329)$. In the complex process of causality, some causal factors are indeed "universals" because they are present in all cases of both the occurrence and the nonoccurrence of a phenotype. According to Gifford's example, the absence of a $1000^{\circ} \mathrm{C}$ temperature is a necessary condition for having a given condition. But it does not constitute an explanation of this particular condition, since human life in general requires that factor. On the contrary, a genetic effect is "specific" if the modification of a gene has some effect on the considered phenotype but not on other ones. Moreover, in Gifford's account, this phenotype must be individuated (i.e. correspond to one precise unit of description), neither too broad nor too narrow. Hypercholesterolemia for instance, cannot be considered a genetic disease because one can distinguish familial and sporadic forms of this disease.

Even if Gifford's account of genetic disease has been criticized, it constitutes a good criterion to resolve the causal selection problem. To be sure, it is not an absolute criterion because for some diseases there are many necessary conditions which, properly construed, are neither universal nor specific. Nonetheless, it constitutes a clear echo of the conceptualization of genetic disease analyzed in the first section of the present article. The concept of specific effect refers indeed to molecular genetics, and Gifford explains that one should accord less importance to the question of knowing if a trait is genetic than to the steps genetic variations modify in biological pathways.

\subsection{The epidemiological strategy}

The second relevant approach to genetic causality refers to populations rather than individuals. It states that a disease is genetic with respect to a population $\mathrm{P}$ if genetic factors make the difference between the persons who suffer from the disease and the rest of the P population (see Smith, 2007). In this epidemiological account of genetic disease, it is entirely possible that the analysis of one population will label a disease "genetic" while a similar analysis of another population will label the disease "environmental". Philosophical discussions often refer to the example of a water supply contaminated by a pathogen. Only half the villagers fall ill and the researchers assume that their genes confer some resistance to the pathogen. On the scale of the village, the infection is genetic because genes are practically sufficient to bring about the condition. On the planetary scale however, the disease would probably be labeled "environmental" and the pathogen considered as the cause of the disease.

Relativity is thus the key word for this conceptualization of genetic disease, and some scholars reject this strategy for solving the causal selection problem because the concept of genetic disease becomes relative to population. After all, the problem of causal selection is simply pushed back to the problem of population selection. Moreover, this epidemiological account of genetic disease is completely at a loss when faced with the task of explaining individual occurrences of a disease. Again, the attempt to give an absolute criterion to label a disease as genetic ends in failure.

\subsection{A pragmatic solution to the causal selection problem}


Reflecting on the differences between genetic and genomic medicines and on the role of geneticists in the personalized medicine of the future, C. Epstein stated that even if the genetics of common diseases is more complex than the genetics of monogenic conditions, it is "still genetics nonetheless" $(2006,437)$. The fact that genomic tools make it possible to acquire the knowledge required to identify the genetic components of these diseases, as well as the fact that genetic testing procedures may be carried out to predict who is at risk, constitute for him significant arguments.

More generally, the pragmatic position states that a cause makes the difference when it is the most "manipulable". The above example of the water supply shows that we consider the pathogenic agent to be the main cause of the disease because we believe that we can manipulate this cause: we can clean the water supply for instance. However, practical as well as theoretical arguments can be opposed to the position that gene factors could be the main cause of genetic diseases.

Until now, genetic manipulations are not the easiest way to manage diseases. Difficulties in finding safe methods to introduce a therapeutic gene into human cells as well as ethical problems regarding human genetic manipulation are strong impediments to gene therapy advances. Even if gene transfer protocols have been approved for human use in inherited diseases, cancers and common diseases like blindness, hemophilia, thalassemia, melanoma or Parkinson's disease (see the "Gene Therapy Clinical Trial Worldwide" database), gene therapy is still an exception and the "manipulability" cannot be considered as a solid criterion for the causal selection problem.

Should this pragmatic approach thus be reduced to its preventive dimension? In that case, a disease would be labeled "genetic" when a genetic test could be put on the market which predicts a relative risk of developing the condition. This position falls under a more general objection: given the role played by the genes in all traits, any phenotype will be genetically manipulable in principle. However, according to our knowledge of the etiology of phenotypes, any trait that is genetically manipulable in principle will also be environmentally manipulable in principle. So the causal selection problem reappears, this time in a pragmatic guise: how does it benefit us to describe a disease as genetic?

\section{4. The problem of geneticization}

Some philosophers are still working hard to resolve the causal selection problem, but one can also ask what the consequences of giving up on that problem would be.

As medical researches led to an extension of the concept of genetic disease, a neutral concept of geneticization was proposed. This neutral concept holds that the process of geneticization consists in a change in the explanation of a disease such that it is considered to take place at the molecular level. Some sociologists have proposed local and precise descriptions of that change for particular diseases such as cystic fibrosis or diabetes (Hedgecoe, 2002 and 2003; Keer, 2004 and 2005).

The difficulties in finding a satisfactory solution to the problem of causal selection may however lead to a much more critical position. In the 1990s, some sociologists and philosophers (Lippman, 1991; Nelkin and Lindee, 1995; Hoedemaekers and Ten Have, 1998) described the rapid expansion of genetics in medicine and society, which they called "geneticization" in a critical sense. They stated that there were ideological reasons for favoring genetic explanations of differences in abilities, behavior and disease. For them, labeling a disease as genetic refers more to a set of social and 
political commitments about the best way to allocate resources than to biological or medical considerations. Till today, the concept of geneticization is still associated with deterministic and reductionist descriptions and interpretations of human life and behavior. It also underlines how using genomic vocabulary and metaphors may have perverse social consequences. Indeed, the confusion of "genetic" and "inevitable" favors individual responsibility instead of costly public policies for protecting people. Furthermore, it leads to the belief that the only solution to genetic problem is the selection of individuals based on genetic criteria. Eugenics is not far off.

The problem of causal selection may have no solution as long as philosophers continue to seek a unique and absolute criterion that could justify holding a genetic factor to be the main cause of a complex biological phenomenon such as disease. However, the three above mentioned strategies constitutes good points of reference when it comes to the question of whether it is justified to label a particular condition as genetic (see also Dekeuwer, 2015). This section nonetheless also highlights what is perhaps a more difficult problem than the causal selection one: that a viable criterion of genetic disease may not be sufficient to hold back a dangerous process of geneticization.

\section{Interactionism and new perspectives in medicine}

This last part endorses the causal parity principle (also called "causal democracy" principle) and considers the interactionist attempts to deal with genetic factors involved in human diseases. According to this principle, there is no reason to give any special privilege to genetic causality. Two versions of interactionism that maintain this principle open new perspectives when it comes to conceptualizing genetic diseases.

\subsection{From genetic predisposition to interactive predisposition}

Research into the determinism of biological traits typically regards the phenotype as the product genes on the one hand and the environment (i. e. everything else) on the other. They inquire how the phenotype varies as the DNA sequences are held constant and other factors change. The genotype's reaction norm - a very common tool in biology - is the graphic representation of this strategy.

P. Kitcher (2003) showed that this tool is scientifically valid and respects the principle of causal parity. Indeed, isolating certain causal factors by holding them constant in order to see how the effect varies when other factors are modified is justified. Following the principle of causal parity, it is then possible to study phenotype variation as a function of a genotype held constant when environmental factors change. This is how most biological and medical research operates. But it would be also possible to carry out a study in which an environmental factor would be held constant and the variation of phenotypes would be observed as a function of this factor. In this case, we could see that for a single genotype, the phenotype varies as a function of the environmental factor. Kitcher underlines that if both designs are congruent with the principle of causal democracy and pertinent for studying the determinism of a complex trait, scientists often use the reaction norm in a genocentric way, showing how keeping a genotype constant in various environments leads to an invariable phenotype.

This genocentrism may lead to misleading views about genetic determinism and has urged some scholars to carefully and critically investigate biological and medical studies of complex traits, as did 
J. Tabery (2009). In a 2002 study, A. Caspi et al. studied an interaction between a gene controlling neuroenzymatic activity (MAOA), exposure to maltreatment in childhood and antisocial personality disorder (ASPD). What made this article so interesting is the fact that the authors found a particular genetic factor and a particular environmental factor which, when joined, were highly correlated to a mental disorder. However, as these results have been reported in the media as the discovery of a "Murder Gene" and the interaction conceptualized as a genetic predisposition to violence, Tabery established a meticulous distinction between genetic predisposition and interactive predisposition. He also underlined the ethical consequences of the misconstruction of the concept of genetic predisposition to violence.

At first glance, the reaction norms published by Caspi et al. show that individuals with high-MAOA activity gradually increase their risk of developing ASPD as incidents of childhood maltreatment increase. On the contrary, individuals with low-MAOA activity drastically increase their risk of developing ASPD as incidents of childhood maltreatment increase. This was interpreted as showing a genetic predisposition to ASPD.

According to Tabery's definition, a genetic predisposition consistently increases the risk of developing the phenotype in each tested environment. In the case of a genetic predisposition to ASPD, individuals from the low-MAOA activity group would maintain their relatively elevated risk for ASPD in every tested environment (none, probable, severe maltreatment in childhood). Moreover, the genetic difference between the low-MAOA activity and the high-MAOA activity groups would consistently put individuals from the low-MAOA activity group at an increased risk of developing ASPD compared to the individuals from the high-MAOA activity group. However, the reaction norms from the two groups clearly cross over. The graph shows a change between the two groups across the different environments: in the absence of childhood maltreatment, the high-MAOA activity group scored higher than the low-MAOA activity group. In other words, high-MAOA activity is protective or aggravating, depending on the environment. Thus, Tabery elaborates the concept of interactive predisposition, which refers to the presence of a genetic difference between various groups that "both increases and decreases the probability of individuals from one group, in comparison to individuals from the other group(s) developing a particular phenotypic trait depending on the environmental conditions experienced" $(2009,35)$.

This work constitutes a good example of an attempt to perfect the much used conceptualization of genetic predisposition. It respects the principle of causal parity while pointing out the ethical and social risks of conceptual misconstruction. It thus shows how further studies employing an interactionist perspective could improve concepts originating from genetics.

\subsection{The concept of developmental interaction}

Since the work of R. Lewontin and S. Oyama, the Developmental System Theory (DST) has tried to understand the development of biological organisms from an interactionist point of view. But, far from improving classical concepts, it rejects some funding biological notions. For DST, the assumptions that are required to build the reaction norm, for instance the genotype-phenotype distinction, must be reconsidered. 
DST has the merit of drawing attention to some conceptual difficulties involved in contemporary studies. The partition between "gene" and "environment" for instance is very fuzzy. This is not only because the gene is difficult to define as a concept (see Beurton et al. 2000). It is also because one cannot bring together into the same category epigenetics, internal environment (the cell functioning for example), external environment (altitude, exposition to a pathogen and so on) and social environment. If not on the assumption of genocentrism, how could the partition between genes on the one hand and "all the rest" on the other be justified? Indeed, it is only by incorporating many more layers of structures and processes than the gene-environment interaction that we may account for the processes of development, including diseases.

DST also contests the idea that the partition between genetic influence and environmental influence could be quantitatively assessed. The biometric concept of interaction, introduced by Ronald Fisher, defines genetic and environmental influences statistically. Consequently, biometric studies yield only statistical and not biological evidence for genetic influence. On the contrary, interaction should be conceptualized as the result of differences in unique developmental combinations of a particular set of factors that have to be identified.

For DST, the development of an organism - and diseases are each particular instances of the development of an organism - has to be understood as a co-constructionist process. Coconstructionist interactionism emphasizes that an organism constructs its environment, just as the environment constructs the organism. An organism inherits resources from its environment and modifies it. That modified environment in turn may modify gene regulations as well as the distribution of genetic variants in the population. In this kind of dynamic approach, development at each stage builds on the results of developments at earlier stages.

DST offers important criticisms of other approaches in biology, but can it lead to a major shift in the conceptualization of genetic diseases? If this theory has the major advantages of respecting the causal democracy principle as well as carrying out its explanations at the level of the organism, its lack of applications has been criticized. However, even if DST seems a long way from contemporary medical perspectives, network medicine appears today to be quite close to the basic project of DTS.

\subsection{The concept of genetic disease in the context of network medicine}

The expression "network medicine" was introduced by Albert-László Barabási in a 2007 publication entitled "Network medicine: from obesity to the "diseasome"" published in the New England journal of medicine. Its key hypothesis is that a disease is rarely a consequence of an abnormality in a single gene, but reflects various biological processes that interact in a complex network. A disease reflects the perturbations of a series of linked networks that incorporate several intracellular as well as extracellular components. Yet biological systems are not random and their many components are connected in complicated ways that can be characterized by a core set of organizing principles.

Using network theory, it is possible to study biological systems by presenting biological factors like molecules or diseases as nodes and their relationships (for instance physical interaction or shared gene) as edges. Network medicine focuses mainly on topology to study the networks that are at play in the development of diseases. For instance, it uses metabolic networks to study protein-protein interactions, but also social networks to map the spreading of disease across populations. 
The human interactome is supposed to reflect the whole set of interactions that occur in a healthy human organism. Today, it encompasses protein-protein interaction networks, metabolic networks, regulatory networks and RNA networks (see Barabási et al., 2011). For instance, in metabolic networks, nodes are metabolites that are linked if they participate in the same biochemical reaction. The human disease network or diseasome represents disease maps, whose nodes are diseases and whose links represent molecular relationships between for instance shared genes or shared metabolic pathways. These maps bring into light disease modules, each disease module being a group of interacting components (DNA, RNA, transcription factors, metabolites and so on) which, if disrupted, results in a pathological phenotype.

Leroy Hood developed the idea that network medicine could lead to 4P medicine: a preventive, predictive, personalized and participatory medicine (this $4 \mathrm{P}$ medicine is also called " personalized medicine " and refers to the project analyzed above in the first section). In reality, however, network medicine is more challenged by its various limitations than it is successful in revolutionizing medical practices. Among these limitations, one can recall that many factors affecting disease modules remain unknown, but also the limitations of statistical tools used to explore the role of networks in disease (see Barabási, 2011). Several advances regarding the conceptualization of genetic disease in network medicine are nevertheless worth mentioning.

Network medicine studies are an essential complement of the GWAS, as they lean on their results to map networks, which in turn may lead to the identification of new genetic factors involved in pathological phenotypes. For instance, the underlying disease module of a specific disease is likely to include all disease-modifying genes involved in epigenetic, transcriptional or regulatory phenomena. Thus network medicine can guide further experimental work towards uncovering disease mechanisms as well as their complementary genetic factors. For instance, Y. Chen et al. (2008) identified a network of genes from mouse liver and adipose tissues for which three variants associated with obesity and diabetes have been identified.

Using the interactome and the diseasome is also useful to classify diseases (Loscalzo et al., 2007). For instance, mapping interactions of cellular components can lead to the identification of deeper functional or molecular relationships among apparently distinct phenotypes. Diseases could thus be classified not by their differences in phenotype but by their belonging to a specific disease module.

Above all, network medicine research involves a massive change in the definition of genetic disease. Scientists used indeed to seek the genetic factors involved in all diseases, each of them being defined by a specific phenotype. A genetic theory of diseases is now arising: "understanding diseases in the context of these network principles allows us to address some fundamental properties of the genes that are involved in disease. Indeed, only about $10 \%$ of human genes have a known disease association. Thus, do disease genes have unique, quantifiable characteristics that distinguish them from other genes?" (Barabási, 2011, 57). Evidences from genomic research can be used to list the genetic variants associated with each disease. But they can also be used to understand the general principle that links a set of specific genes to the phenomenon of disease. In that context, human disease genes are understood as the genes involved in disease in general. In other words, the concept of genetic disease is in the process of becoming related to a specific category of genes.

Unlike DST, network medicine does not give up on the most common biological distinctions (like the genotype-phenotype distinction for instance). However, its hypotheses are grounded in an 
interactionist perspective, if not a co-constructionist one. They provide a useful framework, taking complexity into account and paving the way for redefining the concept of human disease genes. In that respect, network medicine may offer a satisfactory solution to the above mentioned problem of the triviality that arises from the over-extension of the concept of genetic disease.

\section{Conclusion}

Genomic researches lead to the identification of ever greater numbers of genetic variants involved in human diseases, and especially common diseases. It does not seem to be the case, however, that researchers working on the genetics of complex diseases are trying to show that asthma and bipolar disorder for instance are genetic diseases. Nevertheless, the increasing complexity of our understanding of genotype-phenotype relationships, as well as the fact that virtually every medical condition, disease susceptibility or response to treatment is caused, regulated or influenced by genes contributes to the puzzle of how to conceptualize genetic diseases. Moreover, as the causal selection problem has not received any entirely satisfactory solution, one cannot be satisfied with the conceptualization of genetic disease as a disease mainly caused by one or more identified genes.

To avoid this uncomfortable situation, various paths are possible. The insufficiencies of the conceptual solutions to the causal selection problem may lead one to consider that the concept of genetic disease is only pragmatic. Then, the first option consists in a philosophical or sociological criticism of the process of geneticization that characterizes contemporary medicine, and perhaps also society. The second option gives up on the causal selection problem and follows the principle of causal parity. Considering the interactions between genes and environment, it abandons simple views of gene action and tries to formalize the complex interactions that explain pathogenesis. The distinction between the concept of genetic predisposition and the concept of genetic interaction is an example of such a strategy. In this context, the opposition between environmental and genetic diseases disappears, and, conversely, the specificity of the genes involved in human diseases comes to the fore. With network medicine, conceptualization of genetic disease has begun to move from an attempt to identify genes involved in particular conditions to the study of a specific class of genes with specific biological properties.

Whether it is called "personalized medicine" or "4P medicine", medical researchers share the dream of a preventive medicine based on genetic knowledge. Be it our future or not, the potential social consequences of the misuses of the concepts of genetic disease and genetic predisposition should draw our attention to how cautiously we should be in setting up new routine medical practices. 


\section{Bibliography}

Badano JL, Katsani N (2002) Human genetics and disease: Beyond mendel: an evolving view of human genetic disease transmission. Nature Reviews Genetics 3 (10): 779-790

Barabási AL (2007) Network medicine: from obesity to the "diseasome". New England Journal of Medicine 357: 404-407

Barabási AL, Gulbahce N, Loscalzo J (2011) Network medicine: a network-based approach to human disease. Nature Reviews Genetics 12 (1): 56-68

Bates GP (2005) History of genetic disease: the molecular genetics of Huntington disease - a history. Nat Rev Genet 6 (10): 766-773

Beurton P, Falk R, Rheinberger HJ (eds.) (2000) The Concept of the Gene in Development and Evolution. Historical and Epistemological Perspectives, Cambridge University Press, Cambridge

Braun P, Rietman E, Vidal M (2008) Networking metabolites and diseases. Proceedings of the National Academy of Sciences 105 (29): 9849-9850

Caspi A, McClay J, Moffitt TE et al (2002) Role of genotype in the cycle of violence in maltreated children. Science 297: 851-854

Chen Y, Zhu J, Lum PY et al (2008) Variations in DNA elucidate molecular networks that cause disease. Nature 452: 429-435

Chen S, Parmigiani G (2007) Meta-analysis of BRCA1 and BRCA2 penetrance. Journal of Clinical Oncology 25 (11): 1329-1333

Dausset J (1972) Correlation between histocompatibility antigens and susceptibility to illness. Progress in Clinical Immunology 1: 183-210

Dausset J (1996) Predictive medicine. Eur J Obst Gynaecol Reprod Biol 65: 29-32

Dekeuwer C (2015) Defining genetic disease. In: Huneman P, Lambert G, Silberstein M (eds)

Classification, Disease and Evidence. Springer, Dordrecht, p 147-164

Dipple KM, McCabe ERB (2000) Modifier genes convert "simple" Medelian disorders to complex traits. Molecular genetics and metabolism 71: 43-50

Epstein C J (2006) Medical genetics in the genomic medicine of the 21st century. American Journal of Human Genetics 79(3): 434-438

Friebel TM, Domchek SM, Rebbeck TR (2014) Modifiers of cancer risk in BRCA1 and BRCA2 mutation carriers: systematic review and meta-analysis. J Natl Cancer Inst 106(6): 1-15

Galas DJ, Hodd L (2009) Systems Biology and Emerging Technologies Will Catalyze the Transition from Reactive Medicine to Predictive, Personalized, Preventive and Participatory Medicine.

Interdisciplinary Bio Central 1 (2): 1-4 
Gannett $L$ (1999) What's in a cause? The pragmatic dimensions of genetic explanations. Biol Philos 14: $349-374$

Gifford F (1990) Genetic traits. Biology and philosophy 5 (3): 327-347

Goh KI, Cusick ME, Valle D et al (2007) The human disease network. Proceedings of the National Academy of Sciences 104 (21): 8685-8690

Harper PS (2004) Landmarks in medical genetics: Classic papers with commentaries. Oxford University Press (Oxford monographs on medical genetics), Oxford, New-York, $n^{\circ} 51$

Harper PS (2008) A short history of medical genetics. Oxford University Press (Oxford monographs on medical genetics), Oxford, New-York, n 57

Hedgecoe Adam M (1998) Geneticization, medicalisation and polemics. Medicine, Health Care and Philosophy 1: 235-243

Hedgecoe AM (2002) Reinventing diabetes: classification, division and the geneticization of disease. New Genetics and Society 21 (1): 7-27

Hedgecoe AM (2003) Expansion and uncertainty: cystic fibrosis, classification and genetics. Sociology of Health \& Illness 25 (1): 50-70

Hesslow G (1984) What is a genetic disease? On the relative importance of causes. In: Nordenfelt L, Lindahl BIB (eds) Health, disease, and causal explanations in medicine. Springer, Dordrecht, p 183193

Hesslow G (1988) The problem of causal selection. In: Hilton DJ (ed) Contemporary science and natural explanation: commonsense conceptions of causality. Harvester Press, Brighton, $p$ 11-31

Hoedemaekers R, Ten Have HAMJ (1998) Geneticization: the Cyprus paradigm. Journal of medicine and philosophy 23 (3): 274-287

Hood L (2004) Systems biology and the New Technologies Enable Predictive and Preventative Medicine. Science 306 n5696: 640-643

Hood L, Flores M (2012) A Personal View on Systems Medicine and the Emergence of Proactive P4 Medicine: Predictive, Preventive, Personalized, and Participatory. New Biotechnology 29 (6): 613-624

Kerr A (2004) Giving up on geneticization: a comment on Hedgecoe's "Expansion and uncertainty: cystic fibrosis, classification and genetics". Sociology of health \& illness 26 (1): 102-106

Kerr A (2005) Understanding genetic disease in a socio-historical context: a case study of cystic fibrosis. Sociology of Health \& Illness 27 (7): 873-896

Kitcher P (2003) Battling the undead. How (and how not) to resist genetic determinism. In Kitcher P (ed) In Mendel's mirror. Philosophical reflections on biology. Oxford University Press, Oxford, p283300

Lippman A (1991) Prenatal genetic testing and screening: constructing needs and reinforcing inequalities. American Journal of Law and Medicine 17: 11-50 
Lewontin RC (2000) The Triple Helix: Gene, Organism and Environment. Harvard University Press Cambridge, MA

Loscalzo J, Kohane I, Barabási AL (2007) Human disease classification in the postgenomic era: a complex system approach to human pathobiology . Molecular systems biology 3 (1): 1-11

Magnus D (2004) The concept of genetic disease. In: Caplan AL, McCartney JJ, Sisti DA (eds) Health, disease, and illness: concepts in medicine. Georgetown University Press, Washington, p 233-242

Manolio TA, Collins FS, Cox NJ et al (2009) Finding the missing heritability of complex diseases. Nature 461 no. 7265 : 747-753

Misra S (2013) Human Gene Therapy: A Brief Overview of the Genetic Revolution. JAPI 61: 127-133

Moss L (1992) A kernel of truth: on the reality of the genetic program. In: Proceedings of the philosophy of science association 1992, 1:335-348

Nelkin D, Lindee MS (1995) The DNA Mystique: The Gene as a Cultural Icon. University of Michigan Press, Ann Arbor

Norell S (1984) Models of causation in epidemiology. In Nordenfelt L, Lindahl BIB (eds) Health, disease, and causal explanations in medicine, Dordrecht, Reidel

Oyama S (1985) The ontogeny of information: Developmental systems and evolution. Cambridge University Press, Cambridge, New York

Reich DE, Lander ES (2001) On the allelic spectrum of human disease. Trends in Genetics 17 (9): $502-$ 510

Smith GD (2007) Towards an Adequate Account of Genetic Disease. In: Kincaid H, McKitrick J (eds) Establishing Medical Reality - Essays in the Metaphysics and Epistemology of Biomedical Science. Springer, Dordrecht, p 83-110

Tabery J (2007) Biometric and developmental gene-environment interactions: Looking back, moving forward ». Development and Psychopathology 19 (4): 961-976

Tabery J (2009) From a Genetic Predisposition to an Interactive Predisposition: Rethinking the Ethical Implications of Screening for Gene-Environment Interactions. Journal of Medicine and Philosophy 34 (1): $27-48$

Visscher PM, Brown MA, McCarthy MI et al (2012) Five Years of GWAS Discovery. The American Journal of Human Genetics 90 (1): 7-24

The Welcome Trust Case Control Consortium (2007) Genome-wide association study of 14,000 cases of seven common diseases and 3,000 shared controls. Nature 447: 661-678

Zhou X, Menche J, Barabási AL et al (2014) Human symptoms-disease network. Nature communications 5: 1-10 


\section{Online resources}

Gene Therapy Clinical Trial Worldwide. http://www.abedia.com/wiley/. Accessed 20 July 2015

Online Inheritance in Man. http://omim.org/. Accessed 20 July 2015

\section{Key words}

Causal parity, causal selection, developmental interaction, developmental system theory, genetic disease, genetic medicine, genetic predisposition, geneticization, genocentrism, Genome-Wise Association Study, genomic medicine, genotype-phenotype relation, interactionism, molecular genetics, network medicine, personalized medicine.

\section{Definition of key terms}

Dominant autosomal inheritance: concerns the transmission of a gene that is not localized on sexual chromosome $\mathrm{X}$ or $\mathrm{Y}$. Each parent gives one copy of each gene, which is called an "allele". When a trait is dominant, only one copy is required for the trait to be observed. When both alleles are necessary the mode of inheritance is said to be recessive.

The "central dogma" of molecular biology: this expression refers to a model formulated in 1956 by Francis Crick and republished in Nature in 1970, stating that DNA makes RNA and RNA makes proteins.

Epigenetic: epigenetic phenomena refer to a set of heritable DNA modifications, as for example methylation. They change the expression of genes but do not involve any modification in the underlying DNA sequences. Prader-Willi syndrome for instance is related to epigenetic modifications of DNA.

Genetic linkage study: linkage analysis relies on the co-segregation of a phenotype and specific genetic variants in families to localize a marker statistically associated with the disease.

Genome-Wise Association Study: GWAS consists in scanning markers across genomes of many people to find genetic variations associated with a particular disease. For example, the Welcome Trust Case Control Consortium published in 2007 a GWAS that identified genetic factors for Crohn's Disease, Rheumatoid arthritis, bipolar disorder, coronary artery disease, and types 1 and 2 diabetes.

Heritability: the portion of phenotype variance in a population attributable to genetic factors. This statistical measurement comes from the quantitative genetics introduced by Ronald Fisher.

Human Genome Project: HGP was an international project aimed at determining the human DNA sequence, identifying and mapping the human genes. It started in the $90 \mathrm{~s}$ and was declared complete in 2003. 
Monogenic disease: a monogenic disease is considered as mainly caused by one gene, for example thalassemia, Cystic fibrosis or Huntington Disease. It is transmitted according to Mendel's laws.

SNP: the Single Nucleotide Polymorphism markers are very frequent variations of one nucleotide distributed along the DNA. After the achievement of GWAS studies, association studies used CNVs (Copy Number Variations) markers but the results were rather disappointing.

Variable expressivity: one talks about variable expressivity when the same genetic variant is correlated to different clinical phenotypes.

\section{Summary points}

Extending the concept of genetic disease too far empties the term of its precision and utility, running the risk that it no longer conveys any useful information, as virtually all diseases are genetic in the sense that genetic factors are at work in their development.

Genetic medicine is today a normalized practice based on the knowledge of very high correlations between the presence of specific genetic variants and the apparition of certain diseases in a family.

GWAS, like other association studies formerly practiced, is a black-box strategy. For this reason, a genetic variant that has been identified by association study cannot be considered a cause of a disease.

The contemporary, somewhat anarchical use of the concept of genetic disease is neither the consequence of the ever more complex analyses of the human genome nor the reflection of the extension of our knowledge of the genetic determinants of diseases.

For some sociologists and philosophers, labeling a disease as genetic refers more to a set of social and political commitments about the best way to allocate resources than to biological or medical considerations.

The problem of causal selection may have no solution as long as philosophers will seek a unique and absolute criterion that could justify the choice of a genetic factor as the main cause of a complex biological phenomenon such as disease.

Network medicine provides a useful framework, taking into account complexity and paving the way for redefining the concept of human disease genes as a set of specific genes involved in disease in general. 